\title{
Percussive Augmenter of Rotary Drills (PARoD)
}

\author{
Mircea Badescu, Yoseph Bar-Cohen, Stewart Sherrit, Xiaoqi Bao, \\ Zensheu Chang, Chris Donnelly, and Jack Aldrich \\ Jet Propulsion Laboratory, California Institute of Technology, Pasadena, CA
}

\begin{abstract}
Increasingly, NASA exploration mission objectives include sample acquisition tasks for in-situ analysis or for potential sample return to Earth. To address the requirements for samplers that could be operated at the conditions of the various bodies in the solar system, a piezoelectric actuated percussive sampling device was developed that requires low preload (as low as $10 \mathrm{~N}$ ) which is important for operation at low gravity. This device can be made as light as $400 \mathrm{~g}$, can be operated using low average power, and can drill rocks as hard as basalt. Significant improvement of the penetration rate was achieved by augmenting the hammering action by rotation and use of a fluted bit to provide effective cuttings removal. Generally, hammering is effective in fracturing drilled media while rotation of fluted bits is effective in cuttings removal. To benefit from these two actions, a novel configuration of a percussive mechanism was developed to produce an augmenter of rotary drills. The device was called Percussive Augmenter of Rotary Drills (PARoD). A breadboard PARoD was developed with a $6.4 \mathrm{~mm}(0.25 \mathrm{in})$ diameter bit and was demonstrated to increase the drilling rate of rotation alone by 1.5 to over 10 times. Further, a large PARoD breadboard with $50.8 \mathrm{~mm}$ diameter bit was developed and its tests are currently underway. This paper presents the design, analysis and preliminary test results of the percussive augmenter.
\end{abstract}

Keywords: Drilling, USDC, Planetary Sampling, piezoelectric actuation, percussive augmenter

\section{INTRODUCTION}

Percussion and rotation have long been identified as effective methods of penetrating solid materials and formations [Badescu et al., 2007; Bar-Cohen and Zacny, 2009]. Percussion is very effective at fracturing hard, brittle materials like concrete, rocks and ceramics, whereas rotation is more effective on soft and/or ductile materials such as wood, plastics, and ductile metals. A key advantage of rotation is the removal of cuttings from the borehole so the end-effector of the bit continuously comes in contact with fresh surface on the drilled medium. Percussion fractures the material, but continues to hammer at the loose fragments in the borehole unless they are removed. This excessive hammering wastes energy that could be more efficiently used by fracturing the medium. Combining rotation and percussion produces a highly effective penetration mechanism. Existing hammer-drills produce their hammering action mechanically. A novel rotary drill augmenter was developed by the JPL's Advanced Technology Group (under a contract with Placidus LLC) by introducing ultrasonic vibrations onto a rotating bit. The device uses a piezoelectric actuator to augment any rotary drill with high frequency percussion. There are many advantages to using piezoelectric actuators to create hammering [Bar-Cohen and Zacny, 2009; Bao et al., 2003; Bar-Cohen 1999]. They can be operated under low average power using duty cycling. They do not require high axial loading to penetrate materials, making them very easy to use particularly in planets with low gravity. They generate minimal vibration back into the mounting fixtures or the user, making their operation more convenient and less tiring. It is important to note that low power and low applied force are desirable characteristics for sample acquisition in planetary exploratory missions with very tight power budgets, particularly on low gravity planets. The ease of use and quiet operation (compared to other hammer drills) are traits that interest the construction and remodeling industry and the tested augmenter was developed as a prototype for potential commercialization.

Generally, piezoelectric materials generate a charge when under an applied stress. The converse is also true, where an applied electric field produces mechanical strain in piezoelectric materials. By driving the piezoelectric material with a sinusoidal electric field at ultrasonic frequency vibrations with micron size amplitudes are generated. A significant advantage of using piezoelectric stacks as actuators is the ability to operate them at extreme temperatures. This would be particularly of interest to exploration missions to such planets as Titan and Europa where the temperature is as low as $180^{\circ} \mathrm{C}$ and Venus where it is as hot as $460^{\circ} \mathrm{C}$ [Sherrit et al., 2005]. Constraining a piezoelectric stack between a backing and a variable cross-section horn amplifies the vibrations at the horn tip and maintains the PZTs in compression [Sherrit 
et al., 2001; Chang et al., 2004]. The vibrations of the horn are transferred to a drill bit that impacts the drilled media and fractures the formation. A computer program that combines $\mathrm{LabView}^{\mathrm{TM}}$ and MATLAB ${ }^{\mathrm{TM}}$ code was developed to control the drive frequency of the augmenter. To maintain the augmenter in resonance a hill climb algorithm was developed [Aldrich et al., 2006]. The rotation and percussion power are supplied separately allowing the use of various power combinations to test different possibilities and determine the benefit of the augmenter over operating the drill as rotary only. The development of the augmenter was done in two phases. In the first phase a unit with a $6.4 \mathrm{~mm}$ diameter bit was fabricated. In the second phase a new unit was developed with a bit diameter of $50.8 \mathrm{~mm}$. The test results for the prototype with the small bit as well as the preliminary measurements made for the large diameter augmenter are reported in this manuscript.

\section{DESIGN AND ANALYSIS}

The developed augmented drill was designed to operate as a drilling unit with two independent actuators: a rotary actuator and a piezoelectric transducer. The rotation is provided by a commercial drill and it was used to reduce the development costs and demonstrate the prototype augmenter capability to operate as an add-on component for future commercialization. The prototype was called "Percussive Augmenter of Rotary Drills (PARoD)" and two versions of the design were developed - a small diameter and a larger diameter bits, each with a different rotary actuator and piezoelectric transducer. The design of the small diameter version is shown in Figure 1 where the bit is a full face concrete bit with a $6.4 \mathrm{~mm}$ diameter crown. It consists of a rotary actuator, a piezoelectric actuator and a drill bit, where the rotary actuator and the bit are off-the-shelf components and the piezoelectric actuator was developed for this reported study. A series of intermediate components were procured or fabricated and integrated into the design including a slip ring for powering the piezoelectric actuator (Figure 1). The component details of the drill are shown in Figure 2 and they include piezoelectric actuator housing, adaptor between the rotary drill chuck and piezoelectric actuator, the slip ring collar and adaptor between the drill bit and piezoelectric actuator horn. The drill bit adaptor design parameters were adjusted such that the drill bit tip reached the largest displacements at the resonant frequency of the augmenter.

To determine the PARoD design parameters and to predict its performance, finite element models were created by using the commercially available tool ANSYS ${ }^{\mathrm{TM}}$. The actuator consists of a piezoelectric stack of four PZT rings that are $40.6 \mathrm{~mm}$ diameter and $5 \mathrm{~mm}$ thick (Figure 3). The actuator design requires that the nodal plane coincides with the mounting plane, so the vibration transmitted to the rotary drill will be minimized at this location. As the ultrasonic actuator is axisymmetric and since we are only interested in the axial modes of resonance, ANSYS axisymmetric elements were used to model half of the cross-section of the transducer. Compared to a 3D model, this 2D model using the axisymmetric elements greatly reduced the size of the FEM model and computation time. Thus, it allowed us to perform a greater number of analyses in order to optimize the design. The resonance frequencies of the actuator also needed to be predicted and a modal analysis was performed to derive the mode shapes, nodal planes, and resonance frequencies.

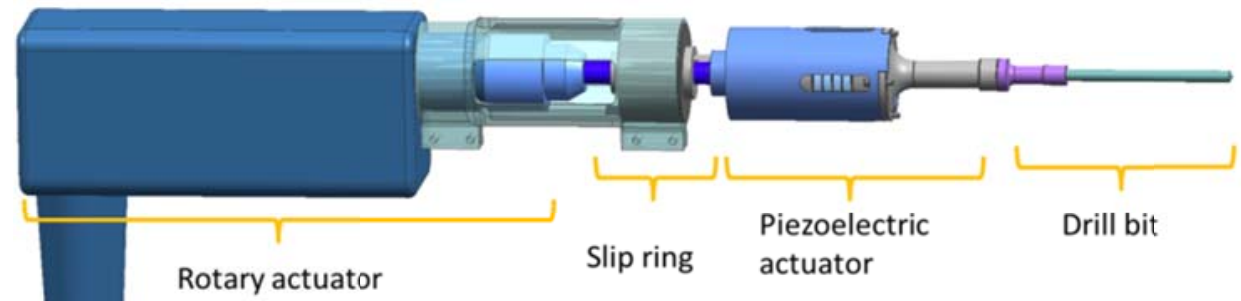

Figure 1: Drill subassemblies

By adjusting the length and diameter of the ultrasonic horn, the nodal plane of the fundamental mode is coincident with the designed mounting plane (Figure 3). The resonance frequency (fr) was predicted to be at $19.24 \mathrm{kHz}$ and the anti-resonance frequency (fa) at $19.5 \mathrm{kHz}$. The electro-mechanical coupling factor $(\kappa)$ was determined by

$$
\kappa=\sqrt{\frac{f_{a}^{2}-f_{r}^{2}}{f_{a}^{2}}}=0.167
$$


After the modal analysis was complete and the design parameters had been determined, harmonic analysis was performed to predict the horn tip displacement and electric power consumption of the actuator. The calculated horn tip displacement vs. frequency curve for $1 \mathrm{~V}$ input voltage is shown in Figure 4 . For $100 \mathrm{~V}$ input voltage, the maximum tip displacement is $7.07 \mu \mathrm{m}$ at $19.2 \mathrm{kHz}$. The power consumption vs. frequency for $1 \mathrm{~V}$ input voltage is shown in Figure 5, where for $100 \mathrm{~V}$ input voltage the maximum peak power is $19.7 \mathrm{~W}$.

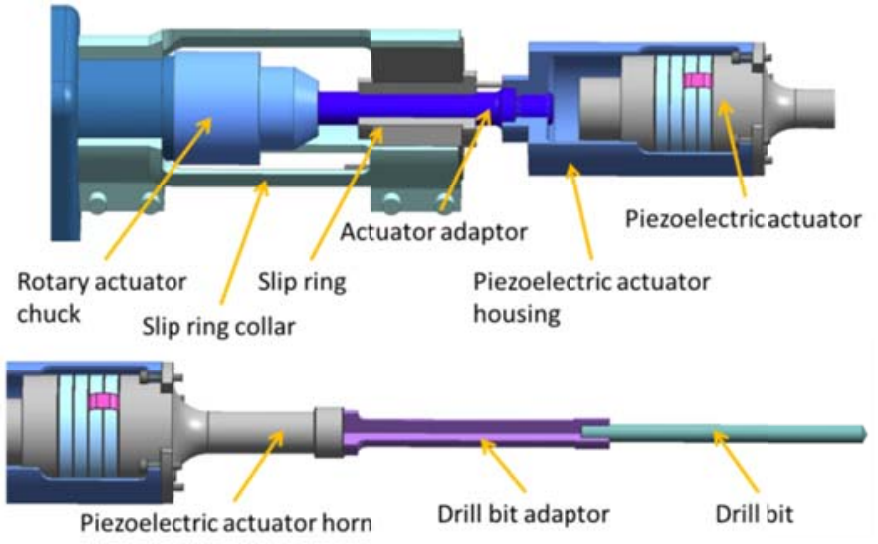

Figure 2: Components details

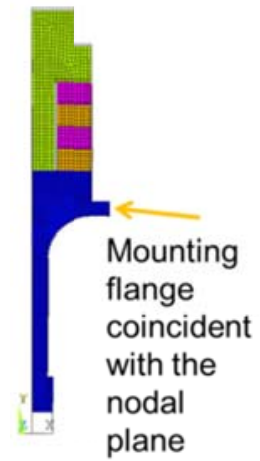

Figure 3: Transducer finite element model

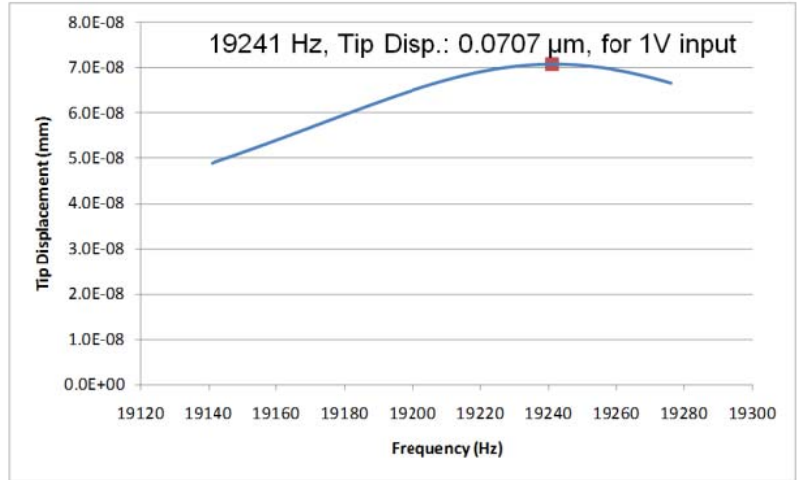

Figure 4: Horn tip displacement vs. frequency (1 V input)

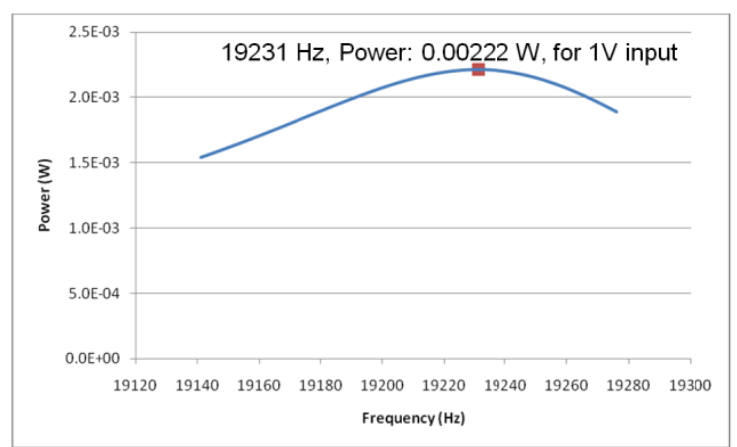

Figure 5: Ultrasonic actuator power vs. frequency for $1 \mathrm{~V}$ input

After the ultrasonic actuator was analyzed, the adaptor and the drill bit were added to the FEM model to establish the model for the complete PARoD design. The length and the diameter of the adaptor were adjusted to make the nodal plane coincide with the mounting plane. Iterations of analysis showed that an adaptor with $80 \mathrm{~mm}$ in length and $10 \mathrm{~mm}$ in diameter can make the nodal plane coincide with the mounting plane. The third axial mode, at $19.7 \mathrm{kHz}$, of the PARoD was determined for the actuator. Its mode shape is shown in Figure 6 and its anti-resonance frequency is 19.8 $\mathrm{kHz}$ which makes the electro-mechanical coupling factor $(\kappa)$ to be 0.09 , as determined using Equation (1). Further harmonic analysis was performed to predict the drill bit tip displacement and electric power consumption of the PARoD. 
The analysis showed that the maximum drill bit tip displacement for $100 \mathrm{~V}$ input voltage at $19.7 \mathrm{kHz}$ is $3.66 \mu \mathrm{m}$, and the peak power is $10.9 \mathrm{~W}$. For the scaled up version of the design (shown in Figures 7 and 8 ) the coring bit diameter is 50.8 $\mathrm{mm}$ and the piezoelectric actuator consist of a stack of 6 PZT rings that are $50.8 \mathrm{~mm}$ diameter and $5 \mathrm{~mm}$ thickness.

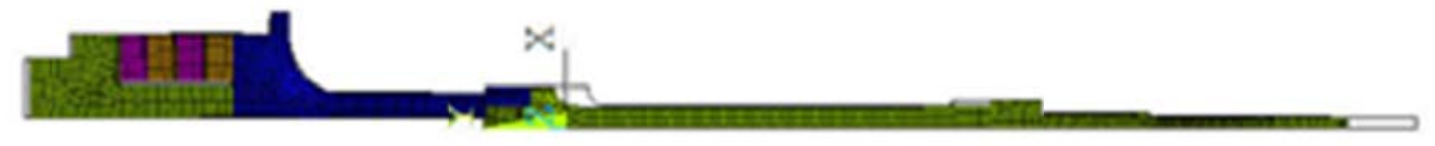

Figure 6: PARoD FEM model

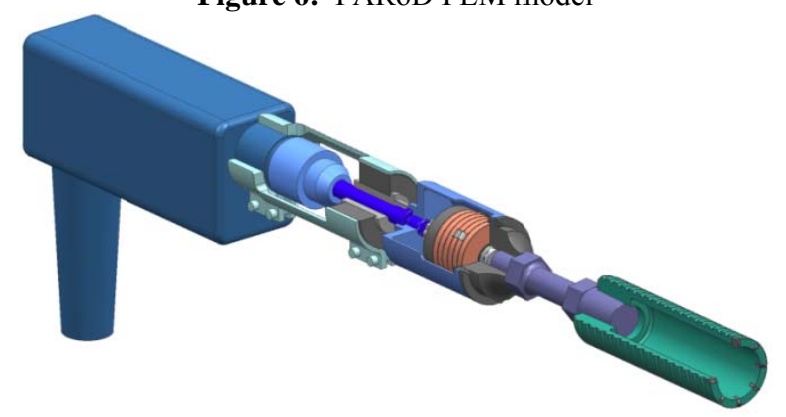

Figure 7 PARoD scaled up version design

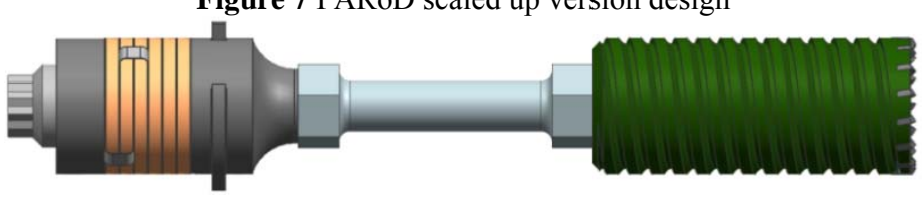

Figure 8 Piezoelectric actuator and coring bit design

\section{FABRICATION AND INTEGRATION}

Both PARoD versions integrated the fabricated parts with commercially available rotary drills. Once they were assembled, they were mounted onto a testbed built specifically for this task that allows for the control of the weight-onbit and maintains the alignment and feed of the drill bit during drilling. The $6.35 \mathrm{~mm}$ drill bit augmenter was fabricated and assembled with a rapid prototyped slip ring collar, aluminum housing for the piezoelectric transducer and a drill bit brazed into the bit adaptor (Figure 9). This smaller bit breadboard allowed the use of a simple linear slide but initially small misalignments were encountered in the drill axis with respect to the sliding axis resulting in induced vibration to the drill and the support stand. The lessons learned from fabricating the small size augmenter and the test stand were implemented into the fabrication of the large scale augmenter (Figure 10). Effectively, every component of the large augmenter was selected or designed to be compatible with the required breadboard, including a related commercial rotary drill and bits, a slip ring that allowed a larger diameter piezoelectric actuator adaptor shaft, and a different method of fabricating the piezoelectric actuator housing. Cast aluminum was used to fabricate a rough housing and then machined to adjust the alignment critical dimensions. The support stand was re-built as a 3D frame with a much sturdier sliding fixture.

The piezoelectric actuator adaptor and housing were fabricated separately to reduce costs but they could be integrated into one part for better augmenter alignment. Similarly, the drill bit, the drill bit adaptor and the ultrasonic horn can be combined into one or two parts depending on the decision to make the augmenter more versatile by allowing more configurations for a given piezoelectric actuator or to increase the efficiency of the augmenter by reducing losses that each interface introduces.

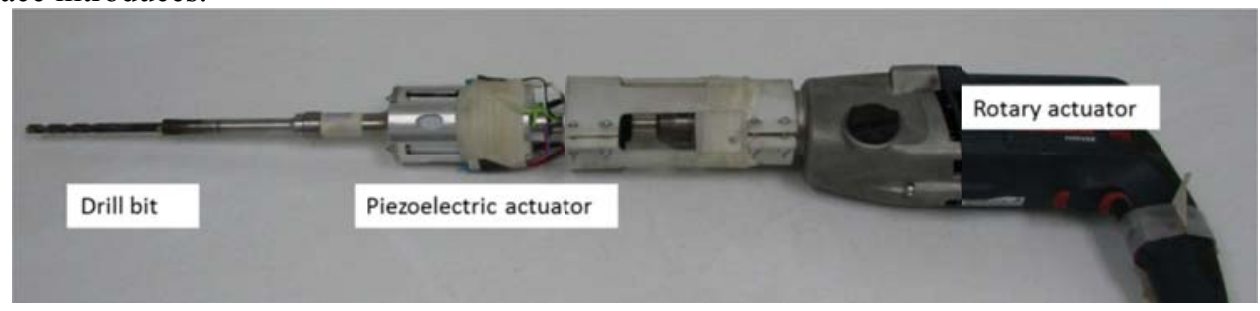

Figure 9: The $6.35 \mathrm{~mm}$ drill bit augmenter 


\section{TESTS OF THE AUGMENTERS}

The test results presented here cover the small diameter augmenter as it was the first to be fabricated and assembled. The large augmenter was assembled recently and is currently being tested with the results to be reported in future publications.

A series of tests in limestone were performed using rotation only, rotation and hammering by the mechanism of the commercial drill, as well as rotation with the hammering by the augmenter. Due to differences in drilling times, all comparisons and charts were recorded to show the drilling rate. Also, all the test data are based on the peak power of the augmenter and not the average power. The average power of the piezoelectric actuator would be less than half of the peak power as the actuator is not driven in resonance continuously.

Two sets of tests were performed. In the first test set the rotary power was kept constant and the augmenter power was increased. In the second set of tests the total power was kept constant. The results of these drilling tests are shown in Figure 11 where the rotary actuator power was kept constant at $50 \mathrm{~W}$ and the augmenter peak or maximum power was increased from zero to $200 \mathrm{~W}$. The upper limit of the driving power was determined by monitoring the heating of the actuator. Also the weight on bit used during the tests had two different values, $4.2 \mathrm{kgf}(41 \mathrm{~N})$ and 7.0 kgf $(69 \mathrm{~N})$. The test results show that the penetration rate increases with the power to the piezoelectric actuator and that the weight on bit has little effect on the drilling rate. Higher weight on bit values were attempted but the flexibility of the support structure did not allow reliable results. It is believed that this flexibility also produced larger variation in the tests results at higher power levels.

Figure 12 and Figure 13 show the results for the condition of constant total power drilling, where the total power for the two actuators was set at $160 \mathrm{~W}$. Tests were done for two values of the

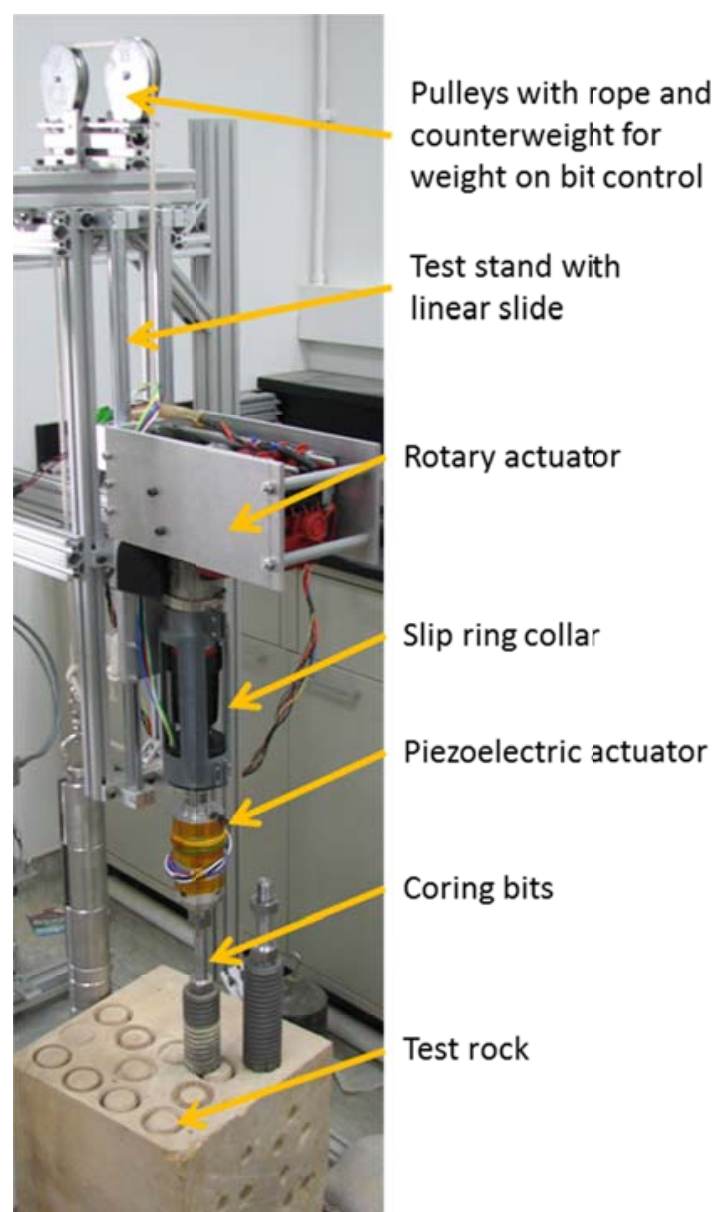

Figure 10: The $50.8 \mathrm{~mm}$ coring bit augmenter mounted onto the test stand/testbed weight on bit: $4.2 \mathrm{kgf}(41 \mathrm{~N}$, Figure 12) and $7.0 \mathrm{kgf}(69 \mathrm{~N}$, Figure 13). The tests for the $7.0 \mathrm{kgf}(69 \mathrm{~N})$ weight on bit were stopped at the power level of $120 \mathrm{~W}$ to the piezoelectric actuator. Overall, it has been observed that as the preload is increased more power to the rotary actuator is required in order to keep the bit turning. Figure 12 shows that the penetration rate for the combination of the augmenter and rotation for the $4.2 \mathrm{kgf}(41 \mathrm{~N})$ weight on bit can be as much as 10 times higher than the penetration rate for rotation alone using the same total power. In this figure, the rotation only is shown as the diamond data points on the vertical axis, where the augmenter power is zero. Further, for these rock and drilling conditions, the penetration rate of combination of the augmenter and rotation is at a higher level of performance than the commercial rotary-hammer drill (the triangle data points in Figure 12).

Figure 13 shows that the penetration rate for the combination of the augmenter and rotation for $7.0 \mathrm{kgf}(69 \mathrm{~N})$ weight on bit is higher than the penetration rate for rotation alone using the same total power. In this figure, the rotation only is shown as the square data points on the vertical axis where it is zero augmenter power. Further, for this rock and drilling conditions, the penetration rate of combination of the augmenter and rotation is at about the same level of performance as the commercial rotary-hammer drill (the round data points in Figure 13). The data scattering for this test case is very large and we believe it could be caused by the problems in bit brazing to the bit adapter and the stiffness of the test stand. These results are the main reason we implemented the sturdier test stand design and fabrication for the larger scale augmenter testing.

Initial tests of the impedance spectra were made on the $50.8 \mathrm{~mm}$ diameter piezoelectric transducer for the large augmenter and a high value of 0.5 of the electromechanical coupling factor (k) for low power was observed (Figure 14). This result suggests the possibility that improved performance will be obtained with the scaled up version of the developed augmenter. 


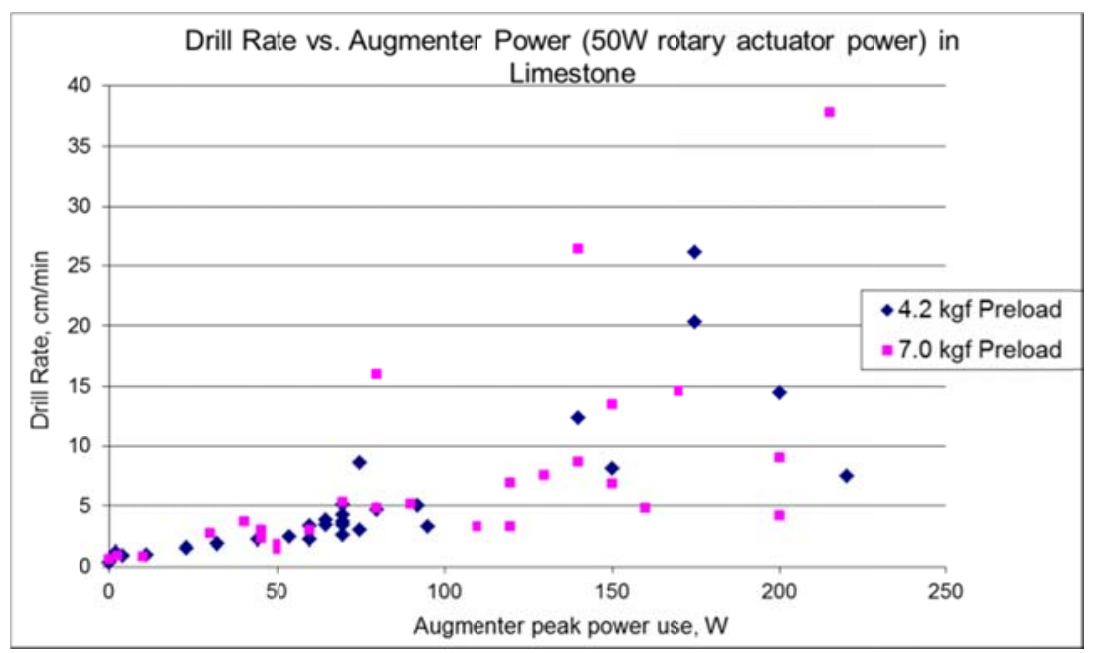

Figure 11: Tests of the small scale augmenter with the rotating bit using constant power to the rotary drill.

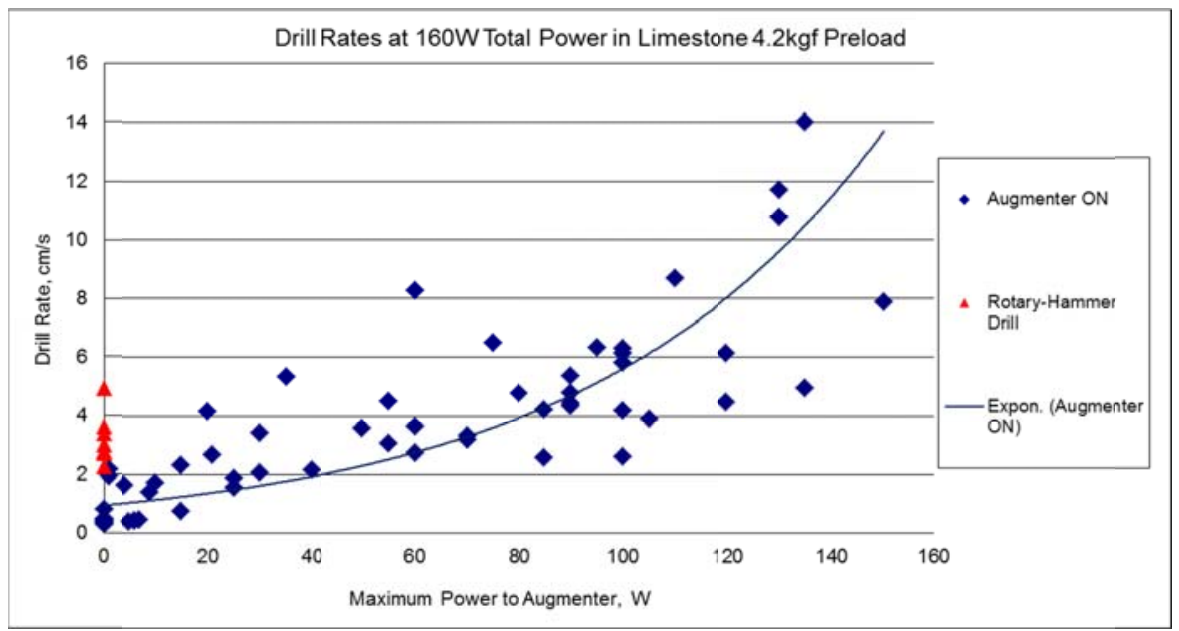

Figure 12: Drilling tests in limestone using constant total power to the rotary and augmenter actuators for $4.2 \mathrm{kgf}(41 \mathrm{~N})$ preload.

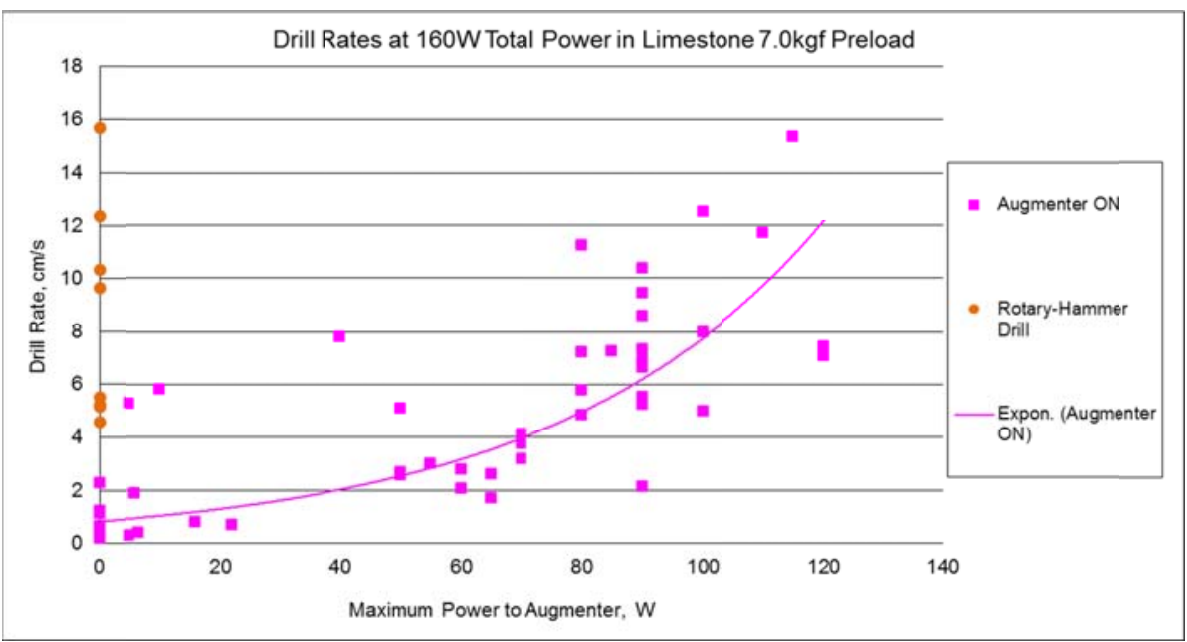

Figure 13: Drilling tests in limestone using constant total power to the rotary and augmenter actuators for $7.0 \mathrm{kgf}(69 \mathrm{~N})$ preload 


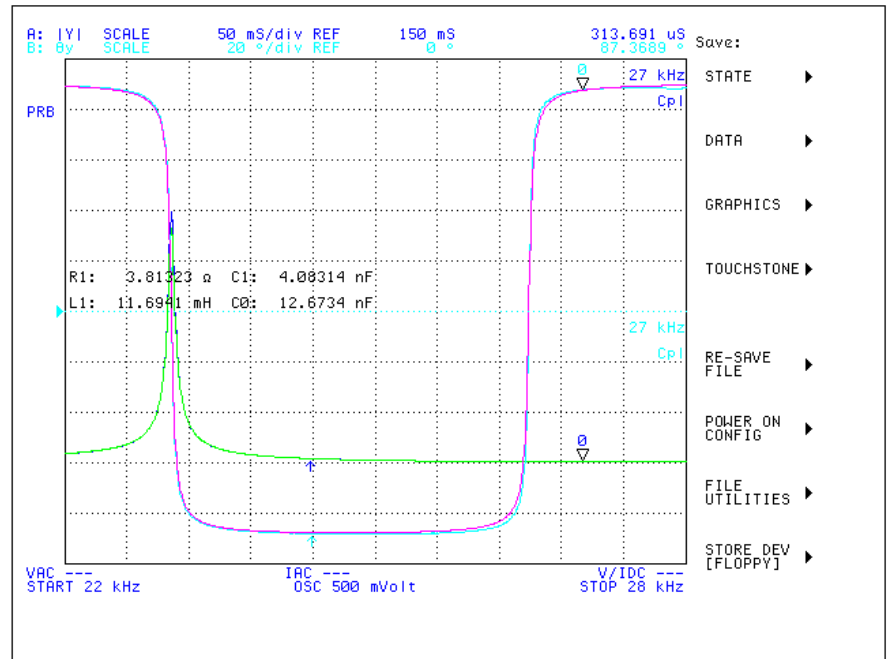

Figure 14: The admittance spectra of the $50.8 \mathrm{~mm}$ diameter PZT rings transducer.

\section{CONCLUSIONS AND FUTURE WORK}

This paper reports the development of an augmenter for rotary drills that is driven by a piezoelectric actuator to induce vibrations onto a rotary bit. The augmenter was conceived to support potential future NASA exploration missions with the objective of acquiring samples for in-situ analytical instruments. Significant improvement of the penetration rate was achieved by the introduction of the ultrasonic hammering action that is generated on fluted bits by the piezoelectric actuator. This improvement is due to the enhanced capability to fracture the rock and the fluidization of the cuttings which aids the removal process. The development was done in two steps where the first step focused on a small scale version with a $6.4 \mathrm{~mm}(0.25 \mathrm{inch})$ diameter bit. The tests of the small scale rotary drill and augmenter over a wide range of power combinations with a total power of $160 \mathrm{~W}$ drilling in limestone have shown drilling penetration rates as good as or better than the commercial rotary-hammer drill. Specifically, it was demonstrated to increase the drilling rate by 1.5 to over 10 times over rotary only drilling. In the second phase that is currently underway, a large version was developed using a bit that is $50.8 \mathrm{~mm}$ diameter. Preliminary results of testing the large design version are quite encouraging and progress will be reported in future publications.

The current design of the augmenter device is made as an attachment to a rotary drill. In future modifications it can be designed and fabricated as a single unit where the augmenter is integrated into a single drill. The integrated augmenter can be mounted along a drill shaft behind the rotary motor eliminating the need for an adaptor and thus providing a more compact packaging.

\section{ACKNOWLEDGEMENT}

Research reported in this manuscript was conducted at the Jet Propulsion Laboratory (JPL), California Institute of Technology, under a contract with the National Aeronautics and Space Administration (NASA). The reported novel Percussive Augmenter of Rotary Drill (PARoD) was developed under a contract with Placidus Placid Technologies LLC, Israel-USA. The authors would like to express their appreciation of the support of Joshua Leavitt, CEO, Placidus Technologies LLC, Israel-USA.

\section{REFERENCES}

[1] Aldrich J., S. Sherrit, M. Badescu Y. Bar-Cohen, X. Bao, and Z. Chang, "Controller using extremum-seeking to drive heavily perturbed electroactive actuators at resonance" NTR Docket No. 43519, (2006).

[2] Badescu M., S. Sherrit, Y. Bar-Cohen, X. Bao, and S. Kassab, "Ultrasonic/Sonic Rotary-Hammer Drill (USRoHD)," NTR Docket No. 44765, (2006).

[3] Bao X., Y. Bar-Cohen, Z. Chang, B. P. Dolgin, S. Sherrit, D. S. Pal, S. Du, and T. Peterson, IEEE Transaction on Ultrasonics, Ferroelectrics and Frequency Control (UFFC), Vol. 50, No. 9, pp. 1147-1160, (2003). 
[4] Bar-Cohen Y. and K. Zacny (Eds.), "Drilling in Extreme Environments - Penetration and Sampling on Earth and Other Planets," Wiley - VCH, Hoboken, NJ, ISBN-10: 3527408525, ISBN-13: 9783527408528, pp. 1-827, (2009).

[5] Bar-Cohen Y., S. Sherrit, B. Dolgin, T. Peterson, D. Pal and J. Kroh, "Smart-ultrasonic/sonic driller/corer," U.S. Patent No. 6,863,136, (2005).

[6] Chang, Z., S. Sherrit, X. Bao, and Y. Bar-Cohen, "Design and Analysis of Ultrasonic Horn for USDC (Ultrasonic/Sonic Driller/Corer)," Proceedings of the SPIE Smart Structures Conference, SPIE Vol. 5388-34, (2004).

[7] Sherrit S., S. A. Askins, M. Gradziel, B. P. Dolgin, Y. Bar-Cohen, X. Bao, and Z. Cheng, "Novel Ultrasonic Horns for power ultrasonics," NTR Docket No. 30489, (2001).

[8] Sherrit, S., "Smart Material/Actuator Needs in Extreme Environments in Space," Proceedings of the SPIE Smart Structures Conference, SPIE Vol. 5761-48, (2005). 